\title{
Prevalence and Covariates of Undiagnosed Hypertension in the Adult Population of Puducherry, South India
}

\author{
Bharati DR¹, Nandi P2, Yamuna TV³, Lokeshmaran A4 , Agarwal L5,Singh JB66, Basu M77, Das P8,Pal R
}

${ }^{1}$ Associate Professor, Department of Community Medicine, Mahatma Gandhi Medical College and Research Institute,Shri Balajee Vidyapeeth,Pillayarkuppam, Puducherry.

${ }^{2}$ Associate Professor, Department of Community Medicine, Mahatma Gandhi Medical College and Research Institute, Shri Balajee Vidyapeeth, Pillayarkuppam, Puducherry.

${ }^{3}$ Sister tutor, Nursing College, Jawaharlal Nehru Institute of Postgraduate Medical Education and Research, Puducherry. ${ }^{4}$ Tutor-cum-statistician, Department of Community Medicine, Mahatma Gandhi Medical College and Research Institute, Shri Balajee Vidyapeeth, Pillayarkuppam, Puducherry.

${ }^{5}$ Assistant Professor, Department of Community Medicine, Mahatma Gandhi Medical College and Research Institute, Shri Balajee Vidyapeeth,Pillayarkuppam, Puducherry.

${ }^{6}$ Tutor, MGM Medical College and LSK Hospital, Kishanganj, Bihar.

${ }^{7}$ Associate Professor, Department of Community Medicine, Postgraduate Institute of Medical Education and Research, Kolkata.

${ }^{8}$ Associate Professor, Department of Community Medicine, Medical College, Kolkata.

${ }^{9}$ Professor, Community Medicine, Sikkim Manipal Institute of Medical Sciences, 5th Mile, Tadong, Gangtok, Sikkim, India.

\section{Original Article}

\section{Corresponding Author:}

Dr. Ranabir Pal, M.D

Professor, Community Medicine, Sikkim Manipal Institute of Medical Sciences, 5th Mile, Tadong,

Gangtok, Sikkim, India-737 102

Email: ranabirmon@yahoo.co.in

\section{Abstract}

\section{Background}

Globally the prevalence of both hypertension and prehypertension are increasing and pre-hypertension has been associated with higher cardiovascular risk. The objective of this study was to find out the prevalence of undiagnosed hypertension and pre-hypertension and associated covariates among adults of rural and urban area of Puducherry.

\section{Material and Methods}

A population based cross-sectional study was carried out in the field practices area of Department of Community
Medicine, Mahatma Gandhi Medical College and Research Institute without intervention.

\section{Results}

Among 856 participants, 27.6 percent were hypertensive and 57 percent pre-hypertensive; the magnitude of both stage 1 and stage 2 hypertension increased with the age. Of the hypertensives, 26.3 and 28.7 percent were from rural and urban area respectively, whereas in pre-hypertension the corresponding figures were 61 and 53.4 percent. Significantly increased risk of hypertension was noted among adults with increasing age, having less than six members in the family, suffering from obesity (general and abdominal obesity) and living sedentary life. On the other hand, the probability of having increased risk of prehypertension was significantly higher among adults living in the rural areas, having non-vegetarian dietary habit, suffering from obesity (general and abdominal obesity), living sedentary life and having family history of hypertension. Multivariate logistic regression however showed significant correlation of hypertension with increasing age, sedentary lifestyle, tobacco addiction and obesity (general and central). Significant correlates of prehypertensive were rural residence, sedentary living habits, 
family history of hypertension, tobacco addiction and obesity (general and central).

\section{Conclusion}

The present findings show high prevalence of hypertension and pre-hypertension among population of Puducherry in South India.

\section{Key Words}

Pre-hypertension, Hypertension, Covariates.

\section{Background}

Hypertension is a major contributor to the global morbidity burden with devastating downstream outcomes with heavy financial burden on scarce health resources ${ }^{1}$. Hypertension is reported to be the fourth contributor to premature death in developed countries and the seventh in developing countries $^{2}$. According to Kearney, worldwide reports indicate that nearly 1 billion adults (more than a quarter of the world's population) had hypertension in 2000 with a prevalence rate of 26.4 percent, and this is predicted to increase to 1.56 billion by 2025 and a prevalence rate of 29.2 percent $^{3}$. The prevalence rates in India are now almost comparable to those in the USA ${ }^{4}$. Prevalence of HTN in people aged $\geq 20$ years by world region and gender in 2000 and 2025 showed that in India in 2000 the combined urban and rural prevalence was 20.6 percent among males and 20.9 percent among females and in 2025 the projected rate will be 22.9 percent among males and 23.6 percent among females ${ }^{3}$. Pre-hypertension also has been associated with higher cardiovascular risk and is estimated to decrease the average life expectancy by as much as five years, because pre-hypertension often develops into hypertension $(50 \%$ of people within 4 years) ${ }^{5,6}$.

Even then, high blood pressure (hypertension) is still largely ignored as a public health problem in most developing countries; because of the asymptomatic nature of the condition, most hypertensives are unaware they are affected $^{1,7}$ and so many people with hypertension do not seek the help of a doctor. Therefore, the detection and control of hypertension is a major public health challenge both developed and developing countries.

Various factors might have contributed to this rising trend, and, among the hypotheses, the consequences of urbanization, such as changes in life style patterns, diet, and stress and the overall epidemiologic transition, India is experiencing currently. As hypertension is one of the most important modifiable risk factors for cardiovascular disease, epidemiological studies to assess the prevalence of pre- hypertension and hypertension are urgently needed in developing countries like India to determine the baseline against which future trends in risk factor levels can be assessed and preventive strategies planned to promote health among all sections of the populations including reducing obesity, increasing physical activity, decreasing the extra salt intake of the population and a concerted effort to promote awareness about hypertension, its risk factors, and risk behaviours ${ }^{4,7}$.

Researchers from the Departments of Endocrinology \& Pathology, Sanjay Gandhi Postgraduate Institute of Medical Sciences of Lucknow in India, noted an age and sex adjusted prevalence of hypertension was 32.2 per cent and prehypertension was 32.3 per cent among adults aged 30 years and above in an urban colony of high-income group residents in the city of Lucknow, north India; hypertension was highest in the age group 60-69 years (64\%) and prehypertension was highest (36\%) in the group $30-39$ years ${ }^{8}$.

With these perspectives, this study was conducted to find out the prevalence of hypertension and pre-hypertension among adults 30 years and above with the covariates associated with hypertension among the adult population of rural and urban area of Puducherry, South India.

\section{Material and Methods}

\section{Settings and design:}

A population based cross-sectional study was carried out in the field practice area of Department of Community Medicine, Mahatma Gandhi Medical College and Research Institute, Pondy-Cuddalore Main Road, Pillayarkuppam, Puducherry - 607402, India Department of Community Medicine, Mahatma Gandhi Medical College and Research Institute without intervention among adults, aged 30 years and more.

\section{Study period:}

The study was conducted from $22^{\text {nd }}$ June 2009 to $31^{\text {st }}$ December 2009.

\section{Sample size and sampling design:}

With the available studies relating to prevalence of hypertension in India, the prevalence of 24.9 percent was taken for estimating the sample size requirement with limit of accuracy as 5 percent alpha error, 3 percent absolute allowable error, 15 percent for non-response and a design effect of 10 percent, accordingly the minimum sample size required for the study was found to be 1040 individuals ${ }^{9}$. All eligible individuals from selected area were identified from electoral roll of election commission of India, followed by preparation of two separate lists of eligible, one for urban 
area and another for rural area and there after 524 subjects from rural area and 516 subjects from urban area were selected by random sampling method. 184 data (nonresponder / on treatment) were discarded and finally 856 data (410 data from rural area and 446 data from urban area) were analyzed.

\section{Study instrument:}

The data collection tool used for the study was an interview schedule that was developed at the Institute with the assistance from the faculty members and other experts. This pre-designed and pre-tested questionnaire contained questions relating to the information on family characteristics like residence, number of members in the family, family history of hypertension, and personal characteristics like age, sex, education, occupation, dietary habit, alcohol consumption and smoking habit. By initial translation, back-translation, re-translation followed by pilot study the questionnaire was custom-made for the study. The pilot study was carried out at the institute among general subjects following which some of the questions from the interview schedule were modified. Blood pressures as well as anthropometric measurements were taken.

\section{Data collection procedure:}

All the participants were explained about the purpose of the study and were ensured strict confidentiality. Informed consent was obtained from all the participants. Blood pressure measurement was done for each participant after half an hour of sitting in any calm area and due explanation to the examined participant about the objective of the study. The blood pressure was measured using the auscultatory method with a standardized calibrated mercury column type sphygmomanometer and an appropriate sized cuff encircling at least 80 percent of the arm in the seated posture, with feet on the floor and arm supported at heart level. Following a standardized protocol, they made two separate measurements and recorded the average of the two measurements after proper rest. In some cases, where high blood pressure was recorded for the first time, the physicians checked the blood pressure more than twice and took the average of the two close readings. Systolic BP is the point at which the first of 2 or more sounds is heard (phase I) and diastolic BP is the point before the disappearance of sounds (phase 5). Data regarding family and personal characteristics were recorded by personal interview. Body weight was measured (to the nearest $0.5 \mathrm{~kg}$ ) in the standing motionless on the bath room scale with feet $15 \mathrm{~cm}$ apart, and weight equally distributed on each leg. Height was measured (to the nearest $0.5 \mathrm{~cm}$ ) by stadeometer in standing position with closed feet, holding their breath in full inspiration and Frankfurt line of vision. Waist circumference was measured by flexible nonstretchable measuring tape in standing. A maximum of three visits were conducted for those who could not be contacted during the first visit.

\section{Diagnostic criteria of undiagnosed Hypertension:}

Seventh Report of the Joint National Committee on Prevention, Detection, Evaluation, and Treatment of High Blood Pressure (JNC 7), 2004 recommendation was used for diagnostic criteria for the diagnosis of hypertension "Persons with the systolic blood pressure $\geq 140 \mathrm{~mm}$ of $\mathrm{Hg}$ and or diastolic blood pressure $\geq 90 \mathrm{~mm}$ of $\mathrm{Hg}$ and not on treatment were classified as undiagnosed case of hypertension" ${ }^{10}$.

\section{Diagnostic criteria of undiagnosed Prehypertension:}

Seventh Report of the Joint National Committee on Prevention, Detection, Evaluation, and Treatment of High Blood Pressure (JNC 7), 2004 recommendation used for diagnostic criteria for the diagnosis of hypertension "Persons with the systolic blood pressure $\geq 120 \mathrm{~mm}$ of $\mathrm{Hg}$ but $<140 \mathrm{~mm}$ of $\mathrm{Hg}$ and or diastolic blood pressure $\geq 80 \mathrm{~mm}$ of $\mathrm{Hg}$ but $<90 \mathrm{~mm}$ of $\mathrm{Hg}$ and not on treatment were classified as undiagnosed case of pre-hypertension" ${ }^{\prime 9}$.

\section{Diagnostic criteria of obesity:}

Cut-offs level of BMI (general obesity) was taken as follows: Normal: $18.50-24.99 \mathrm{~kg} / \mathrm{m} 2$, Overweight (Pre-obese): 25.00 $29.99 \mathrm{~kg} / \mathrm{m} 2$, Obesity: $\geq 30.00 \mathrm{~kg} / \mathrm{m} 2$ according to WHO criteria $^{11}$. We followed cut-offs level of waist circumference (Abdominal obesity) for men $\geq 102 \mathrm{~cm}$, and for women $\geq 88$ $\mathrm{cm}$ the National, Heart, Lung and Blood Institute criteria ${ }^{12}$.

\section{Diagnostic criteria of smoking:}

Current smoker was defined as someone who at the time of study used to smoke either daily or occasionally. Nonsmokers were individuals who never smoked at all.

\section{Diagnostic criteria of alcohol intake:}

A "current drinker" was defined as one who consumed one or more drinks of any type of alcohol in the year preceding the study.

\section{Statistical analysis used:}

Data were analyzed by Epi Info 7 and SPSS version 16th statistical software package. Proportion of adult person with pre-hypertension and hypertension was presented as percentage. Hypertensives were excluded when analyzing pre-hypertensives, and vice versa. Risk factors for hypertension and pre-hypertension were separately tested in a univariate and multiple logistic regression analysis. Independent variables tested were age, gender, residence, number of family members, education, occupation, dilatory 
habit, alcohol and tobacco addiction, family history of hypertension, body mass index and waist circumference. In univariate analysis chi square test was employed for comparison, Odds ratio (OR) and 95 percent confidence interval ( $95 \%$ C.I.) of categorical variables. Backward LR method was used to perform binary logistic regressions, where presence of pre-hypertension and hypertension were used separately as dependent variable while others as independent variables. A two-tailed $p$ value $<0.05$ was considered significant.

\section{Results}

Among 856 participants, 320 (37.4\%) were males and 536 (62.6\%) were females; 236 (27.6\%) had hypertension (systolic blood pressure $\geq 140 \mathrm{~mm}$ of $\mathrm{Hg}$ and or diastolic blood pressure $\geq 90 \mathrm{~mm}$ of $\mathrm{Hg}$ ); 488 (57\%) had prehypertension (systolic $120-139$ or diastolic $80-89 \mathrm{~mm}$ of $\mathrm{Hg}$ ). The magnitude of hypertension increased as age of the study participants increased for both stage 1 and stage 2 hypertension, (except for stage 2 cases, aged more than or equal to 70 years) which was statistically significant. (Table 1)

Table 1: Prevalence of hypertension in different age groups

\begin{tabular}{|c|c|c|c|c|}
\hline \multirow{2}{*}{ Age $(\mathrm{n})$} & Normal & Prehypertensive & \multicolumn{2}{|c|}{ Hypertensive } \\
& & & Stage 1 & Stage 2 \\
\hline $30-39(330)$ & $74(22.4 \%)$ & $218(66.1 \%)$ & $34(10.3 \%)$ & $04(1.2 \%)$ \\
\hline $40-49(208)$ & $16(7.7 \%)$ & $126(60.6 \%)$ & $46(22.1 \%)$ & $20(9.6 \%)$ \\
\hline $50-59(158)$ & $26(16.5 \%)$ & $72(45.6 \%)$ & $40(25.3 \%)$ & $20(12.7 \%)$ \\
\hline $60-69(112)$ & $14(12.5 \%)$ & $48(42.9 \%)$ & $34(30.4 \%)$ & $16(14.3 \%)$ \\
\hline$>=70(48)$ & $02(4.2 \%)$ & $24(50.0 \%)$ & $18(37.5 \%)$ & $04(8.3 \%)$ \\
\hline Total = 856 & $132(15.4 \%)$ & $488(57.0 \%)$ & $172(20.1 \%)$ & $64(7.5 \%)$ \\
\hline
\end{tabular}

Table 2 and 3 show that the hypertensives, 128 (28.7\%) and 108 (26.3\%) were from urban and rural area respectively, whereas in pre-hypertension the corresponding figures were 250 (61\%) and 238 (53.4\%) respectively. The proportion of hypertension was 45.8, 44.6, 38.0, 31.7 and 11.5 percents respectively in persons aged more than or equal to 70 and ranges of 60-69, 50-59, 40-49, 30-39 years; the differences was statistically significant. There were no significant sex difference of prevalence of pre-hypertension and hypertension. In the univariate analysis, probability of having increased risk of hypertension was significantly higher among adults with increasing age, having less than six members in the family, suffering from obesity (general and abdominal obesity) and living sedentary life, on the other hand probability of having increased risk of prehypertension was significantly higher among adults living in the rural areas, non-vegetarian dietary habit, obesity (general and abdominal obesity), sedentary lifestyle and family history of hypertension.

Table 2: Correlates of systemic hypertension: Univariate Analysis

\begin{tabular}{|c|c|c|c|c|c|c|c|}
\hline \multirow{2}{*}{ Variables } & \multirow{2}{*}{$\begin{array}{l}\text { Normal } \\
\text { No. }(\%)\end{array}$} & \multicolumn{3}{|c|}{ Hypertensive } & \multicolumn{3}{|c|}{ Pre-Hypertensive } \\
\hline & & No. (\%) & $\begin{array}{c}\text { OR } \\
\text { (95\% C.I.) }\end{array}$ & $\begin{array}{c}P \\
\text { value }\end{array}$ & No. (\%) & $\begin{array}{c}\text { OR } \\
(95 \% \text { C.I.) }\end{array}$ & \begin{tabular}{|c}
$\mathrm{P}$ \\
value
\end{tabular} \\
\hline \multicolumn{8}{|c|}{ Residence } \\
\hline $\begin{array}{l}\text { Rural } \\
\text { (410) }\end{array}$ & $\begin{array}{c}52 \\
(12.7)\end{array}$ & $\begin{array}{c}108 \\
(26.3)\end{array}$ & 1 & & $\begin{array}{c}250 \\
(61.0)\end{array}$ & 1 & \\
\hline $\begin{array}{l}\text { Urban } \\
(446)\end{array}$ & $\begin{array}{c}80 \\
(17.9)\end{array}$ & $\begin{array}{c}128 \\
(28.7)\end{array}$ & $\begin{array}{c}0.77 \\
(0.45-1.18)\end{array}$ & $0.24 \times$ & $\begin{array}{c}238 \\
(53.4)\end{array}$ & $\begin{array}{c}0.62 \\
(0.48-0.91)\end{array}$ & $0.02^{*}$ \\
\hline \multicolumn{8}{|c|}{ Age } \\
\hline $\begin{array}{l}30-39 \\
(330)\end{array}$ & $\begin{array}{c}74 \\
(22.4)\end{array}$ & $\begin{array}{c}38 \\
(11.5)\end{array}$ & 1 & & $\begin{array}{c}218 \\
(66.1)\end{array}$ & 1 & \\
\hline $\begin{array}{l}40-49 \\
(208)\end{array}$ & $\begin{array}{c}16 \\
(7.7)\end{array}$ & $\begin{array}{c}66 \\
(31.7)\end{array}$ & $\begin{array}{c}8.03 \\
(4.10-15.72)\end{array}$ & & $\begin{array}{c}126 \\
(60.6)\end{array}$ & $\begin{array}{c}2.67 \\
(1.49-4.79)\end{array}$ & \\
\hline $\begin{array}{l}50-59 \\
(158)\end{array}$ & $\begin{array}{c}26 \\
(16.5)\end{array}$ & $\begin{array}{c}60 \\
(38.0)\end{array}$ & $\begin{array}{c}4.49 \\
(2.46-8.22)\end{array}$ & $0.0001^{\dagger}$ & $\begin{array}{c}72 \\
(45.6)\end{array}$ & $\begin{array}{c}0.94 \\
(0.55-1.58)\end{array}$ & $0.19 x$ \\
\hline $\begin{array}{l}60-69 \\
(112)\end{array}$ & $\begin{array}{c}14 \\
(12.5)\end{array}$ & $\begin{array}{c}50 \\
(44.6)\end{array}$ & $\begin{array}{c}6.95 \\
(3.41-14.18)\end{array}$ & & $\begin{array}{c}48 \\
(42.9)\end{array}$ & $\begin{array}{c}1.16 \\
(0.60-2.23)\end{array}$ & \\
\hline $\begin{array}{l}\geq 70 \\
(48)\end{array}$ & $\begin{array}{c}2 \\
(4.2)\end{array}$ & $\begin{array}{c}22 \\
(45.8)\end{array}$ & $\begin{array}{c}21.42 \\
(4.78-95.95)\end{array}$ & & $\begin{array}{c}24 \\
(50.0)\end{array}$ & $\begin{array}{c}4.07 \\
(0.94-17.65)\end{array}$ & \\
\hline \multicolumn{8}{|c|}{ Sex } \\
\hline $\begin{array}{c}\text { Female } \\
(536)\end{array}$ & $\begin{array}{c}86 \\
(16.0)\end{array}$ & $\begin{array}{c}150 \\
(28.0)\end{array}$ & 1 & & $\begin{array}{c}300 \\
(56.0)\end{array}$ & 1 & \\
\hline $\begin{array}{l}\text { Male } \\
(320)\end{array}$ & $\begin{array}{c}46 \\
(14.4)\end{array}$ & $\begin{array}{c}86 \\
(26.9)\end{array}$ & $\begin{array}{c}1.07 \\
(0.68-1.67)\end{array}$ & $0.76 \times$ & $\begin{array}{c}188 \\
(58.8)\end{array}$ & $\begin{array}{c}1.17 \\
(0.78-1.75)\end{array}$ & $0.44 \times$ \\
\hline \multicolumn{8}{|c|}{ Education } \\
\hline \begin{tabular}{|c} 
Illiterate \\
$(608)$
\end{tabular} & $\begin{array}{c}96 \\
(15.8 \%)\end{array}$ & $\begin{array}{c}150 \\
(24.7 \%)\end{array}$ & 1 & & $\begin{array}{c}362 \\
(59.5 \%)\end{array}$ & 1 & \\
\hline \begin{tabular}{|} 
Literate \\
$(248)$
\end{tabular} & $\begin{array}{c}36 \\
(14.5)\end{array}$ & $\begin{array}{c}86 \\
(34.7)\end{array}$ & $\begin{array}{c}1.53 \\
(0.96-2.43)\end{array}$ & $0.07 x$ & $\begin{array}{c}126 \\
(50.8)\end{array}$ & $\begin{array}{c}0.93 \\
(0.60-1.43)\end{array}$ & $0.74 x$ \\
\hline \multicolumn{8}{|c|}{ Occupation } \\
\hline $\begin{array}{c}\text { Non- } \\
\text { Sedentary } \\
(314)\end{array}$ & $\begin{array}{c}62 \\
(19.7)\end{array}$ & $\begin{array}{c}74 \\
(23.6)\end{array}$ & 1 & & $\begin{array}{c}178 \\
(56.7)\end{array}$ & 1 & \\
\hline $\begin{array}{c}\text { Sedentary } \\
(542)\end{array}$ & $\begin{array}{c}70 \\
(12.9)\end{array}$ & $\begin{array}{c}162 \\
(29.9)\end{array}$ & $\begin{array}{c}1.94 \\
(1.25-3.00)\end{array}$ & $0.003^{\dagger}$ & $\begin{array}{c}310 \\
(57.2)\end{array}$ & $\begin{array}{c}1.54 \\
(1.04-2.27)\end{array}$ & $0.03^{*}$ \\
\hline \multicolumn{8}{|c|}{ Family size } \\
\hline$<6(568)$ & $\begin{array}{c}78 \\
(13.7 \\
\%)\end{array}$ & $\begin{array}{c}168 \\
(29.6 \%)\end{array}$ & 1 & & $\begin{array}{c}322 \\
(56.7 \%)\end{array}$ & 1 & \\
\hline$\geq 6(288)$ & $\begin{array}{c}54 \\
(18.8)\end{array}$ & $\begin{array}{c}68 \\
(23.6)\end{array}$ & $\begin{array}{c}0.58 \\
(0.37-0.91)\end{array}$ & $0.02 *$ & $\begin{array}{c}166 \\
(57.6)\end{array}$ & $\begin{array}{c}0.74 \\
(0.50-1.10)\end{array}$ & $0.14 \times$ \\
\hline $\begin{array}{l}\text { Total } \\
(856)\end{array}$ & $\begin{array}{c}132 \\
(15.4)\end{array}$ & $\begin{array}{c}236 \\
(27.6)\end{array}$ & & & $\begin{array}{c}488 \\
(57.0)\end{array}$ & & \\
\hline
\end{tabular}

$+p<0.01$, statistically significant

$* p<0.05$, statistically significant

$\times p>0.05$, statistically not significant 
Table 3: Correlates of systemic hypertension: Univariate Analysis

\begin{tabular}{|c|c|c|c|c|c|c|c|}
\hline \multirow{2}{*}{ Variables } & \multirow{2}{*}{$\begin{array}{l}\text { Normal } \\
\text { No. (\%) }\end{array}$} & \multicolumn{3}{|c|}{ Hypertensive } & \multicolumn{3}{|c|}{ Pre-Hypertensive } \\
\hline & & $\begin{array}{l}\text { No. } \\
(\%)\end{array}$ & $\begin{array}{c}\text { OR } \\
(95 \% \text { C.I.) }\end{array}$ & $\begin{array}{c}P \\
\text { value }\end{array}$ & $\begin{array}{l}\text { No. } \\
(\%)\end{array}$ & $\begin{array}{c}\text { OR } \\
(95 \% \text { C.I.) }\end{array}$ & $\mid \begin{array}{c}P \\
\text { value }\end{array}$ \\
\hline \multicolumn{8}{|c|}{ Diet } \\
\hline $\begin{array}{l}\text { Vegetarian } \\
\text { (86) }\end{array}$ & $\begin{array}{c}18 \\
(20.9)\end{array}$ & $\begin{array}{c}32 \\
(37.2)\end{array}$ & 1 & & $\begin{array}{c}36 \\
(41.9)\end{array}$ & 1 & \\
\hline $\begin{array}{l}\text { Non- } \\
\text { Vegetarian } \\
(770)\end{array}$ & $\begin{array}{c}114 \\
(14.8)\end{array}$ & $\begin{array}{c}204 \\
(26.5)\end{array}$ & $\begin{array}{c}1.00 \\
(0.54-1.87)\end{array}$ & $0.98 x$ & $\begin{array}{c}452 \\
(58.7)\end{array}$ & $\begin{array}{c}1.98 \\
(1.08-3.62)\end{array}$ & $0.02 *$ \\
\hline \multicolumn{8}{|c|}{ Family history of hypertension } \\
\hline $\begin{array}{c}\text { Absent } \\
(774)\end{array}$ & $\begin{array}{c}126 \\
(16.3)\end{array}$ & $\begin{array}{c}216 \\
(27.9)\end{array}$ & 1 & & $\begin{array}{c}432 \\
(55.8)\end{array}$ & 1 & \\
\hline $\begin{array}{l}\text { Present } \\
(82)\end{array}$ & $\begin{array}{c}6 \\
(7.3)\end{array}$ & $\begin{array}{c}20 \\
(24.4)\end{array}$ & $\begin{array}{c}1.94 \\
(0.76-4.97)\end{array}$ & $0.16 \times$ & $\begin{array}{c}56 \\
(68.3)\end{array}$ & $\begin{array}{c}2.72 \\
(1.14-6.46)\end{array}$ & $0.02 *$ \\
\hline \multicolumn{8}{|c|}{ Alcohol } \\
\hline $\begin{array}{c}\text { No } \\
(794)\end{array}$ & $\begin{array}{c}124 \\
(15.6)\end{array}$ & $\begin{array}{c}218 \\
(27.5)\end{array}$ & 1 & & $\begin{array}{c}452 \\
(56.9)\end{array}$ & 1 & \\
\hline $\begin{array}{l}\text { Yes } \\
(62)\end{array}$ & $\begin{array}{c}8 \\
(12.9)\end{array}$ & $\begin{array}{c}18 \\
(29.0)\end{array}$ & $\begin{array}{c}1.28 \\
(0.54-3.03)\end{array}$ & $0.57 x$ & $\begin{array}{c}36 \\
(58.1)\end{array}$ & $\begin{array}{c}1.23 \\
(0.56-2.72)\end{array}$ & $0.60 x$ \\
\hline \multicolumn{8}{|c|}{ Tobacco } \\
\hline $\begin{array}{l}\text { No } \\
(776)\end{array}$ & $\begin{array}{c}124 \\
(16.0)\end{array}$ & $\begin{array}{c}208 \\
(26.8)\end{array}$ & 1 & & $\begin{array}{c}444 \\
(57.2)\end{array}$ & 1 & \\
\hline $\begin{array}{l}\text { Yes } \\
(80)\end{array}$ & $\begin{array}{c}8 \\
(10.0)\end{array}$ & $\begin{array}{c}28 \\
(35.0)\end{array}$ & $\begin{array}{c}2.09 \\
(0.92-4.72)\end{array}$ & $0.07 x$ & $\begin{array}{c}44 \\
(55.0)\end{array}$ & $\begin{array}{c}1.53 \\
(0.70-3.34)\end{array}$ & $0.28 x$ \\
\hline \multicolumn{8}{|c|}{ General obesity (BMI) } \\
\hline $\begin{array}{l}<25 \\
(506)\end{array}$ & $\begin{array}{c}96 \\
(19.0)\end{array}$ & $\begin{array}{c}114 \\
(22.5)\end{array}$ & 1 & & $\begin{array}{c}296 \\
(58.5)\end{array}$ & 1 & \\
\hline $\begin{array}{l}\geq 25 \\
(350)\end{array}$ & $\begin{array}{c}36 \\
(10.3)\end{array}$ & $\begin{array}{c}122 \\
(34.9)\end{array}$ & $\begin{array}{c}2.97 \\
(1.87-4.70)\end{array}$ & 0.0001 & $\begin{array}{c}192 \\
(54.9)\end{array}$ & $\begin{array}{c}1.73 \\
(1.13-2.64)\end{array}$ & $0.01 *$ \\
\hline \multicolumn{8}{|c|}{ Central obesity (WC) } \\
\hline $\begin{array}{c}\text { Normal } \\
(384)\end{array}$ & $\begin{array}{c}80 \\
(20.8)\end{array}$ & $\begin{array}{c}78 \\
(20.3)\end{array}$ & 1 & & $\begin{array}{c}226 \\
(58.9)\end{array}$ & 1 & \\
\hline $\begin{array}{l}\text { Obese } \\
(472)\end{array}$ & $\begin{array}{c}52 \\
(11.0)\end{array}$ & $\begin{array}{c}158 \\
(33.5)\end{array}$ & $\begin{array}{c}3.11 \\
(2.00-4.85)\end{array}$ & $0.0001 \dagger$ & $\begin{array}{c}262 \\
(55.5)\end{array}$ & $\begin{array}{c}1.78 \\
(1.20-2.64)\end{array}$ & $\begin{array}{c}0.004 \\
+\end{array}$ \\
\hline $\begin{array}{l}\text { Total } \\
(856)\end{array}$ & $\begin{array}{c}132 \\
(15.4)\end{array}$ & $\begin{array}{c}236 \\
(27.6)\end{array}$ & & & $\begin{array}{c}488 \\
(57.0)\end{array}$ & & \\
\hline
\end{tabular}

$\dagger p<0.01$, statistically significant

$* p<0.05$, statistically significant

$\times p>0.05$, statistically not significant

Table 4 depicts that the significant correlates of hypertension were increasing age, sedentary lifestyle, tobacco addiction and obesity (general and central) by multivariate analysis. Significant correlates of prehypertensive were rural residence, sedentary living habit, family history of hypertension, tobacco addiction and obesity (general and central). The above findings signify that there is likelihood of having increased risk of hypertension was higher among adults with these risk factors in the web of causation.
Table 4: Correlates of systemic hypertension among adults: final model - Bivariate Logistic Regression (backward likelihood ratio method)

\begin{tabular}{|c|c|c|c|c|c|c|c|}
\hline Characteristics & $\begin{array}{l}\text { Regression } \\
\text { Coefficient }\end{array}$ & $\begin{array}{l}\text { Standard } \\
\text { Error }\end{array}$ & Wald & P-value & $\begin{array}{l}\text { Odds } \\
\text { Ratio }\end{array}$ & \multicolumn{2}{|c|}{$\begin{array}{c}95 \% \text { C.I. for } \\
\text { OR }\end{array}$} \\
\hline \multicolumn{8}{|c|}{ Hypertension } \\
\hline Age & 0.074 & 0.011 & 43.71 & $0.0001^{\dagger}$ & 1.08 & 1.05 & 1.10 \\
\hline $\begin{array}{c}\text { Sedentary living } \\
\text { habit }\end{array}$ & 0.631 & 0.259 & 5.94 & $0.015^{*}$ & 1.88 & 1.13 & 3.12 \\
\hline $\begin{array}{l}\text { Tobacco } \\
\text { addiction }\end{array}$ & 1.165 & 0.487 & 5.73 & $0.017^{*}$ & 3.21 & 1.24 & 8.32 \\
\hline $\begin{array}{c}\text { General obesity } \\
\text { (BMI) }\end{array}$ & 0.965 & 0.28 & 11.89 & $0.001+$ & 2.63 & 1.52 & 4.54 \\
\hline $\begin{array}{l}\text { Central obesity } \\
\text { (WC) }\end{array}$ & 0.965 & 0.273 & 12.49 & $0.0001+$ & 2.62 & 1.54 & 4.48 \\
\hline Constant & -8.92 & .268 & 49.49 & $0.0001+$ & 0.00 & & \\
\hline \multicolumn{8}{|c|}{ Pre-Hypertension } \\
\hline Rural residence & -0.541 & 0.21 & 6.67 & $0.010^{*}$ & 0.58 & 0.39 & 0.88 \\
\hline $\begin{array}{c}\text { Sedentary living } \\
\text { habit }\end{array}$ & 0.51 & 0.207 & 6.05 & $0.014^{*}$ & 1.67 & 1.11 & 2.50 \\
\hline $\begin{array}{c}\text { Family history of } \\
\text { hypertension }\end{array}$ & 0.957 & 0.451 & 4.50 & $0.034^{*}$ & 2.61 & 1.08 & 6.31 \\
\hline Tobacco & 0.972 & 0.415 & 5.49 & $0.019 *$ & 2.64 & 1.17 & 5.96 \\
\hline General Obesity & 0.458 & 0.245 & 3.50 & $0.061 x$ & 1.58 & 0.98 & 2.55 \\
\hline Central Obesity & 0.481 & 0.227 & 4.5 & $0.034^{*}$ & 1.62 & 1.04 & 2.52 \\
\hline Constant & -2.052 & 0.915 & 5.03 & $0.025^{*}$ & 0.13 & & \\
\hline
\end{tabular}

$+p<0.01$, statistically significant

$* p<0.05$, statistically significant

$\times p>0.05$, statistically not significant

\section{Discussion}

Our study documented high prevalence of both hypertension and pre-hypertension, and their association with other metabolic and cardiovascular risk factors those are likely to be important contributors to the epidemic of cardiovascular disease among both sexes. Final model of the multivariate logistic regression showed that the important correlates of hypertension were increasing age, sedentary living habit, tobacco addiction and obesity (general and central) and for pre-hypertension rural residence, sedentary living habit, family history of hypertension, tobacco addiction and obesity (general and central). The findings were found to be statistically significant,

Various studies estimated a prevalence rate of hypertension among urban population ranging from 1.24 percent (1949) to 36.4 percent (2003) and for rural people from 1.99 percent (1958) to 21.2 percent (1994). However differential rates are due to different cut off marks in determining the level of hypertension and differing age groups in the study population ${ }^{9}$. While Das et al reported 24.9 percent of hypertension and 35.8 and 47.7 percent pre-hypertension in systolic and diastolic groups respectively in West Bengal, Yadav et al reported 32.2 percent hypertension and 32.3 percent pre-hypertension from Lucknow in the rural Central India, Kannan et al reported 25.2 percent hypertension from 
rural Tamil Nadu, Prashant R et al reported 19.04 percent hypertension and 18.8 percent pre-hypertension from rural central India9, ${ }^{13-15}$. From eastern India, Hazarika et al reported a prevalence of 33.3 percent in the age group of 30 years and above among the native population of Assam in 30 years and above age group ${ }^{16}$. While hypertension was 13 percent in rural adults more than 30 years of age in Jammu, Chow et al reported 27 percent hypertension in rural adults $>30$ years of Andhra Pradesh ${ }^{17,18}$. This was similar to that reported from industrialized economies ${ }^{19-21}$. A study carried out among the Lepchas of Sikkim Himalayas has documented hypertension prevalence of 30.77 per cent among males and 25.77 per cent among females (By using older WHO criteria for hypertension) ${ }^{22}$.The most recent composite national data of the National Nutrition Monitoring Bureau of India has documented hypertension

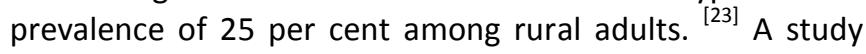
was among the Nicobarese aborigines during 2007 and 2009 noted a 50.5 percent prevalence ${ }^{24}$.

\section{Age}

In the current study, the prevalence of hypertension increased significantly with increasing age but prehypertension has not shown the same trend. Similar findings were reported by different researchers in various parts of India. In a north Indian study, they noted that hypertension was highest in the age group 60-69 years (64\%) and prehypertension was highest (36\%) in the group 30-39 years ${ }^{8,13-15,17,24-27}$. We found comparable reports from other countries also. Erem et al from Turkey and Wang et al from US and Lim et al from the Malaysia noted similar trends $^{20,28,29}$.

\section{Sex}

The prevalence of hypertension was not significantly different among males and females in this study. Others reported that hypertension was not associated with gender differences but significantly higher prevalence of prehypertension was noted in males ${ }^{13,26}$. We found comparable reports from other countries by Erem et al from Turkey ${ }^{28}$. The prevalence of hypertension being significantly higher in females than males were reported by Kanan et al, Prashant et al, Hazarika et al, Raina et al and Malhotra et al ${ }^{14-17,30}$.

\section{Residence}

In this study, magnitude of hypertension was not significantly different among urban area and rural area but pre-hypertension was significantly higher in rural peoples. However other studies have reported higher prevalence in urban areas than rural ${ }^{31,32}$.

\section{Education}

Educational status was not significantly associated with hypertension or pre-hypertension in this study. Educational status showed no association with hypertension in other studies conducted by Manimunda et al, Divan et al and others $^{24,25}$. Erem et al reported from Turkey that lower education was associated with high prevalence of hypertension $^{28}$.

\section{Occupation}

We noted that both hypertension and pre-hypertension was significantly associated with sedentary life style. Prevalence of hypertension was significantly more in people who lead a sedentary life style in the study of Yadav et al, Kokiwar Prashant et al, Singh et al, Malhotra et al, Anand et al, Chockalingam et al and Zachariah et $\mathrm{al}^{13,15,2,30,33-35}$. Similar results have been shown in different ethnic groups ${ }^{36,37}$.

\section{Diet}

In the present study, magnitude of pre-hypertension was significantly more in non-vegetarians than vegetarians but the problem of hypertension was not associated with dietary habit. Reddy et al showed that prevalence of hypertension was more in non- vegetarians ${ }^{26}$.

\section{Family size}

Smaller family size (less than 6 members in the family) showed positive association with hypertension only in univariate analysis and not in multivariate logistic regression analysis $^{38}$. On the contrary, Kannan et al reported that in rural Tamil Nadu, larger family size (more than 6 member) was significantly associated with high prevalence of hypertension $^{14}$.

\section{Family history of hypertension}

Family history of hypertension was significantly associated with pre-hypertension only and not with hypertension in our study. Similar finding was reported by Yadav et al ${ }^{13}$. But positive association of hypertension with family history was reported by Goldstein et al, Carretero et al, Divan et al, Reddy et al and Erem et $\mathrm{al}^{25,26,28,39,40}$.

\section{Alcohol consumption}

In the current study, neither hypertension nor prehypertension was found to have a significant association with alcohol consumption. No association with hypertension was however reported by Divan et al and Gupta et $\mathrm{al}^{25,38}$. Contrarily, KokiwarPrashant et al found a negative association between alcohol intake and hypertension $^{15}$. Others studies have found alcohol consumption to be positively associated with hypertension $^{14,24,26,30}$.

\section{Smoking habit}

In the present study, smoking habit was significantly associated with both hypertension and pre-hypertension. Similar finding was reported by KokiwarPrashant et al, Malhotra et al, Kanan et al , Gupta et al, Reddy et al $^{14,15,26 \text {, }}$ 30, 38 , others reported no association ${ }^{13,25}$.

\section{Body mass index (general obesity)}

We noted that in the participants both hypertension and pre-hypertension were significantly associated with body mass index. Similar findings were reported by Yadav et al, KokiwarPrashant et al, Raina et al, Kanan et al, Gupta et al, 
Manimunda et al,Reddy et al, Raheena et al, Singh et al, Beegom et al and Erem et al ${ }^{13-15,17,24,26-28,38,41}$.

\section{Central obesity}

Central obesity was significantly associated with both hypertension and pre-hypertension in the present study. Similar findings were also reported by other researchers from different parts of India ${ }^{13,15,27,41}$. In a Chennai study, the researchers noted differential risk factors in the analysis in hypertension and pre-hypertension. After the age and sex correction they identified obesity, diet, family history and middle-income group as correlating with pre-hypertension. On the other hand, the factors that predict hypertension were age, sex, smoking, alcohol intake, sedentary lifestyle, and type of work $^{34}$.

The strength of the study was that it was a population based cross-sectional study to find the prevalence of undiagnosed hypertension among adults in both urban and rural areas. So far there has been no study reported in this field in the state and to the extent of our knowledge this was one of the recent studies reported from the South India.

Still there were several limitations that need to be addressed by further research. First, time and the quantity of information relayed to the participants may have rendered them less attentive to the evaluation procedure during interview although participants made no mention of this being a problem. Secondly, we did not evaluate the literacy skills of our participants, but simply evaluated formal education as a risk factor that may have a confounding effect in hypertension and prehypertension. Thirdly, we could not include in the study of the prevalence among adolescents and young adults. Finally, participants in this study were recruited from a corner of country. So they are unlikely to fully represent the diversity within the Indian community itself. This may have lead to a bias favouring the characteristics of the particular groups represented herein, thus limiting the external validity.

\section{Future scope of study}

Future interventions need to strive more to identify the precise genetic and environmental mechanisms underlying increased cardiovascular risk in Indian citizens. Clinical strategies must be developed that identify Indians at increased risk and assess the effectiveness of treatment for insulin resistance, and other cardiovascular risk factors, in this racial group ${ }^{42}$.

\section{Clinical relevance}

Our study suggests that there is a high prevalence of hypertension and pre-hypertension in the population of Puducherry in South India. We hope to find out ways that this study can be repeated at regular intervals in the adjacent areas of the state and in different states of our country by multicentre study to find out a nationally representative database. Our findings had the potential to address the awareness, prevention and intervention gap for hypertension in the high risk population.

\section{What this study adds}

It is a poignant time at present for patients at risk for developing hypertension that is per se a 'silent killer disease'. In our study we observed 27.6 percent hypertensive and 57 percent pre-hypertensive among our participants. Moreover, we noted that in the adults with the increasing age, rural residence, illiteracy, non-vegetarian diet, obesity (general and abdominal obesity), sedentary life and family history of hypertension are more likely hazardous factors for the hypertensive disorders.

\section{Conclusion}

Our findings could lay the foundation for the introduction of primary health care with community participation in combination with lifestyle modification for effective prevention of the cardiovascular diseases. The key factor to prevent hypertension is that we have to generate awareness among our peer groups, public health experts, health services researchers, healthcare providers and planners to consider the higher prevalence and associated risk factors of hypertension as a public health problem in India.

\section{Conflict of Interests}

There are no conflicts of interest among authors arising from the study.

\section{Acknowledgements}

We sincerely thank the medical officers, interns, social workers and nursing health personnel of the Department of Community Medicine of Mahatma Gandhi Medical College and Research Institute for the data collection in the field practices area.

\section{Authors' contributions}

DRB designed the study, deduced the data, drafted the manuscript, and revised it. PN, TVY, Al and LA planned the study, acquired the data, conducted the data analysis, interpreted the data, and revised the manuscript. JBS, NB, PD participated in statistical analysis, interpreted the data, and revised the manuscript. RP critically revised the manuscript. All the authors approved the final document.

\section{References}

1. World Health Organization. Integrated management of cardiovascular risk: Report of a WHO meeting; 9-12 July 2002, Geneva, Switzerland. Geneva: WHO; 2002.pp 7-8.

2. Deepa R, Shanthirani CS, Pradeepa R, Mohan V. Is the 'rule of halves' in hypertension still valid?--Evidence from the Chennai Urban Population Study. J Assoc Physicians India 2003; 51:153-7.

3.Kearney PM, Whelton M, Reynolds K, Muntner P, Whelton PK, He J. Global burden of hypertension: analysis of worldwide data. Lancet 2005; 365: 217-23.

4. Pradeepa R, Mohan V. Hypertension \& pre-hypertension in developing countries. Indian J Med Res 2008; 128(12):688-90. 
5. Chobanian AV, Bakris GL, Black HR, Cushman WC, Green LA, Izzo JL Jr, et al. The seventh report of the Joint National Committee on Prevention, Detection, Evaluation, and Treatment of High Blood Pressure: the JNC7 Report. JAMA 2003; 289(19):2560-72.

6. Russell LB, Valiyeva E, Carson JL. Effects of prehypertension on admissions and deaths: a simulation. Arch Intern Med 2004; 164(19):2119-24.

7. Chandwani $H$, Pandor J, Jivarajani $P$, Jivarajani $H$. Prevalence and correlates of hypertension among adults in the urban area of Jamnagar, Gujarat, India. Electron Physician 2010; 2:52-9.

8. Yadav S, Boddula R, Genitta G, Bhatia V, Bansal B, Kongara $S$, et al. Prevalence \& risk factors of prehypertension \& hypertension in an affluent north Indian population. Indian J Med Res 2008; 128: 712-20.

9. Das SK, Sanyal K, Basu A. Study of urban community survey in India: growing trend of high prevalence of hypertension in a developing country. Int J Med Sci 2005; 2(2):70-3.

10. Seventh report of the Joint National Committee on Prevention, Detection, Evaluation, and Treatment of High Blood Pressure; Hypertension. 2004; 43:e31.

11. BMI Classification [online] 2004 [cited 2012 June 24]. Available from: URL: http://apps.who.int/bmi/index.jsp?introPage=intro_3.html

12. Assessing Your Weight and Health Risk [online] 2006 [cited 2012 March 24]. Available from: URL: http://www.nhlbi.nih.gov/health/public/heart/obesity/lose _wt/risk.html

13. Yadav S, Boddula R, Genitta G, Bhatia V, Bansal B, Kongara $S$, et al. Prevalence \& risk factors of prehypertension \& hypertension in an affluent north Indian population. Indian J Med Res 2008; 128:712-20.

14. Kannan L, Satyamoorthy TS. An epidemiological study of hypertension in a rural household community. Sri Ramachandra Journal of Medicine 2009; 2(2):9-13.

15. KokiwarPrashant R, Gupta Sunil S, Prevalence of hypertension in a rural community of central India. Int J Biol Med Res. 2011; 2(4):950 - 3.

16. Hazarika NC, Narain K, Biswas D, Kalita HC, Mahanta J. Hypertension in the native rural population of Assam. Natl Med J India 2004; 17(6):300-4.

17. Raina DJ, Jamwal DS. Prevalence Study of Overweight/Obesity and Hypertension among Rural Adults. JK SCIENCE 2009; 11(1):20-3.

18. Chow CK, Naidu S, Raju K, Raju R, Joshi R, Sullivan D et al, Significant lipid, adiposity and metabolic abnormalities amongst 4535 Indians from a developing region of rural Andhra Pradesh. Atherosclerosis 2008;196(2):943-52
19. Wenzel D, Sauza JMP, Souza SB. Prevalence of arterial hypertension in young military personnel and associated factors. Rev SaudePubl 2009; 43(5):789-95.

20. Wang Y, Wang QJ. The prevalence of pre-hypertension and hypertension among US adults according to the new joint national committee guidelines: new challenges of the old problem. Arch Intern Med 2004; 164(19):2126-34.

21. Wolf-Maier K, Cooper RS, Banegas JR, Giampaoli S, Hense HW, Joffres $M$, et al. Hypertension prevalence and blood pressure levels in 6 European countries, Canada, and the United States. JAMA 2003; 289(18):2363-9.

22. Mukhopadhyay B. Detecting and preventing hypertension in remote areas. Indian J Med Ethics 2006; 3(4):124-5.

23. Diet \& nutritional status of population and prevalence of hypertension among adults in rural areas. Hyderabad: National Institute of Nutrition; 2006. National Nutrition Monitoring Bureau, National Institute of Nutrition. NNMB Technical Report No 24.

24. Manimunda SP, Sugunan AP, Benegal V, Balakrishna N, Rao MV, Pesala KS. Association of hypertension with risk factors \& hypertension related behaviour among the aboriginal Nicobarese tribe living in Car Nicobar Island, India. Indian J Med Res 2011; 133(3):287-93.

25. Divan V, Chauhan V, Panchal S, Bansal RK. Prevalence of hypertension amongst workers of a fertilizer company in Surat District. Natl J Community Med 2010; 1(2):153-5.

26. Reddy SS, Prabhu GR. Prevalence and risk factors of hypertension in adults in an urban slum, Tirupati, A.P. Indian J Community Med 2005;30(3): 84-6.

27. Singh RB, Beegum R, Ghosh S, Niaz MA, Rastogi V, Rastogi S S, et al. Epidemiological study of hypertension and its determinants in an urban population of North India. J Hum Hypertens 1997; 11(10): 679-85.

28. Erem C, Hacihasanoglu A, Kocak M, Deger O, Topbas M. Prevalence of prehypertension and hypertension and associated risk factors among Turkish adults: Trabzon Hypertension Study. 2009; 31(1):47-8.

29. Lim TO, Morad Z. Prevalence, Awareness, Treatment and Control of Hypertension in the Malaysian Adult Population: Results from the National Health and Morbidity Survey 1996. Singapore Med J 2004; 45(1): 20-7.

30. Malhotra $P$, Kumari $S$, Kumar R, Jain $S$, Sharma BK. Prevalence and determinants of hypertension in an unindustrialised rural population of North India. J Hum Hypertens 1999; 13(7):467-2.

31. Quasem I, Shetye MS, Alex SC, Nag AK, Sarma PS, Thankappan KR. Prevalence, awareness, treatment and control of hypertension among the elderly in Bangladesh and India: a multicentre study. Bull World Health Organ 2001; 79(6):490-500. 
32. Ajay VS, Gupta R, Panniyammakkal J, Chaturvedi V, Prabhakaran D, Reddy KS. National cardiovascular disease database. With support from the Ministry of Health and Family Welfare, Government of India and the World Health Organization. [online] 2012 [cited 2012 June 24]. Available from: URL: http://www.whoindia.org/LinkFiles/NMH_ Resources_National_CVD_database-Final_Report.pdf

33. Anand MP. Prevalence of hypertension amongst Mumbai executives. J Assoc Physicians India 2000; 48(12):1200-1.

34. Chockalingam A, Ganesan N, Venkatesan S, Gnanavelu G, Subramaniam $T$, Jaganathan $V$, et al. Patterns and predictors of prehypertension among "healthy" urban adults in India. Angiology 2005; 56(5):557-63.

35. Zachariah MG, Thankappan KR, Alex SC, Sarma PS, Vasan RS. Prevalence, correlates, awareness, treatment, and control of hypertension in a middle-aged urban population in Kerala. Indian Heart J 2003; 55(3):245-51.

36. Whelton SP, Chin A, Xin X, He J. Effect of aerobic exercise on blood pressure: a meta-analysis of randomized, controlled trials. Ann Intern Med 2002; 136(7):493-503.

37. Bassett DR Jr, Fitzhugh EC, Crespo CJ, King GA, McLaughlin JE. Physical activity and ethnic differences in hypertension prevalence in the United States. Prev Med 2002; 34(2):179-86.

38. Gupta S, Agrawal BK, Sehajpal PK, Goel RK. Prevalence and predictors of essential hypertension in the rural population of Haryana, India: An hospital based study. J Rural Trop Public Health 2011; 10:29 -4.

39. Goldstein IB, Shapiro D, Guthrie D. Ambulatory blood pressure and family history of hypertension in healthy men and women. Am J Hypertens 2006; 19:486-91.

40. Carretero OA, Oparil S. Essential hypertension. Part I: definition and etiology. Circulation 2000; 101: 329-35.

41. Beegom R, Beegom R, Niaz MA, Singh RB. Diet, central obesity and prevalence of hypertension in the urban population of South India. Int J Cardiol 1995; 51(2):183-91.

42. Chambers JC, Kooner JS. Diabetes, insulin resistance and vascular disease among Indian Asians and Europeans. SeminVasc Med 2002; 2(2):199-214.

Article Information

Article history

\begin{tabular}{l|l} 
Received & 15 March 2012
\end{tabular}

Received in revised form 5 April 2012

Accepted

15 April 2012 